\title{
Mesenchymal stem cells stabilize the blood-brain barrier through regulation of astrocytes
}

\author{
Hyun Jung Park ${ }^{1,2}$, Jin Young Shin ${ }^{1,2}$, Ha Na Kim', Se Hee Oh', Sook K. Song ${ }^{3}$ and Phil Hyu Lee ${ }^{1,2^{*}}$
}

\begin{abstract}
Introduction: The blood-brain barrier (BBB) protects the brain against potentially neurotoxic molecules in the circulation, and loss of its integrity may contribute to disease progression in neurodegenerative conditions. Recently, the active role of reactive astrocytes in BBB disruption has become evident in the inflamed brain. In the present study, we investigated whether mesenchymal stem cell (MSC) treatment might modulate reactive astrocytes and thus stabilize BBB integrity through vascular endothelial growth factor A (VEGF-A) signaling in inflammatory conditions.

Methods: For the inflamed brain, we injected LPS using a stereotaxic apparatus and MSCs were injected into the tail vein. At 6 hours and 7 days after LPS injection, we analyzed modulatory effects of MSCs on the change of BBB permeability through VEGF-A signaling using immunochemistry and western blot. To determine the effects of MSCs on VEGF-A-related signaling in cellular system, we had used endothelial cells treated with VEGF-A and co-cultured astrocyte and BV 2 cells treated with lipopolysaccharide (LPS) and then these cells were co-cultured with MSCs.

Results: In LPS-treated rats, MSCs restored Evans blue infiltration and the number of endothelial-barrier antigen (EBA) and P-glycoprotein (p-gp)-expressing cells, which were significantly altered in LPS-treated animals. Additionally, MSC administration following LPS treatment markedly increased the density of astrocytic filaments around vessels and reversed LPS-induced elevations in VEGF-A levels as well as endothelial nitric oxide synthase (eNOS)-dependent downregulation of tight junction proteins in the endothelium. Consequently, MSC treatment reduced neutrophil infiltration and enhanced survival of midbrain dopaminergic neurons in LPS-treated animals. In cellular system, MSC treatment led to a significant reversion of VEGF-A-induced eNOS and tight junction protein expression in endothelial cells, which led to increased EBA expressing cells. Additionally, MSC treatment significantly attenuated LPS-induced increased expressions of IL-1 $\beta$ in microglia and VEGF-A in astrocytes with an increase in IL-10 levels.
\end{abstract}

Conclusion: The present study indicated that MSCs may stabilize BBB permeability by modulating astrocytic endfeet and VEGF-A signaling, which may be relevant to the treatment of Parkinsonian diseases as a candidate for disease modifying therapeutics.

\section{Introduction}

The blood-brain barrier (BBB) tightly regulates ion balance and nutrient transport, and acts as a protective barrier to shield the central nervous system from potentially neurotoxic molecules in the circulation. The integrity of the BBB is altered in neurodegenerative diseases such as Alzheimer's disease and Parkinson's disease (PD), and seems to be influenced by disease severity and duration

\footnotetext{
*Correspondence: phisland@chol.net

${ }^{1}$ Department of Neurology and Brain Research Institute, Yonsei University College of Medicine, 250 Seongsanno, Seodaemun-gu, Seoul 120-752, South Korea

${ }^{2}$ Severance Biomedical Science Institute, Yonsei University College of Medicine, 250 Seongsanno, Seodaemun-gu, Seoul 120-752, South Korea Full list of author information is available at the end of the article
}

$[1,2]$. BBB dysfunction may therefore contribute to disease progression in neurodegenerative conditions, although the underlying mechanism has not been elucidated fully.

Astrocytes, the most abundant cells in the brain, contact the brain vasculature via their endfeet processes. These contacts consist of endothelial tight junctions, which probably prevent diffusion of toxic materials across the BBB [3]. However, reactive astrocytes in the inflamed brain retract their endfeet from vessels, increasing BBB permeability, and proliferate, giving rise to glial scars [4-6]. Furthermore, reactive astrocytes secrete increased levels of the proangiogenic vascular endothelial growth factor A (VEGF-A) more than basal levels in normal physiological conditions. 
Many cellular and in vivo studies have demonstrated that inflammation-induced expression of VEGF-A induces BBB breakdown and immune cell infiltration through disrupted tight junctions, accompanied by altered expression of the tight junction proteins [7-9]. The downstream cascade appears to be mediated by endothelial nitric oxide synthase (eNOS); systemic administration of a selective eNOS inhibitor abrogates VEGF-A-induced BBB disruption and protects against neurologic deficits in models of inflammatory disease [9]. Blockade of VEGFA signaling may therefore be a viable strategy to preserve $\mathrm{BBB}$ integrity in neurodegenerative diseases.

Previously, we demonstrated that mesenchymal stem cells (MSCs) protect dopaminergic neurons through anti-inflammatory properties mediated by modulation of microglial activation in animal models of PD [10, 11]. Additionally, in 1-methyl-4-phenyl-1,2,3,6-tetrahydropyridine (MPTP)-induced PD, Chao et al. [12] reported recently that this effect promoted recovery of BBB integrity. In the present study, we investigated whether MSC treatment might modulate reactive astrocytes and thus stabilize BBB integrity through VEGF-A signaling pathways in both cellular and animal models of inflammatory conditions.

\section{Materials and methods Antibodies}

Antibodies and staining reagents included mouse antityrosine hydroxylase (TH; 1:2000 dilution for brain tissue, dopaminergic neuron marker, Pel-Freez, Rogers, AR, USA) and rabbit anti-ionized calcium-binding adapter molecule 1 (Iba-1; 1:1000 dilution, activated microglia marker, Wako, Osaka, Japan) anti-GFAP (1:1000 dilution, astrocyte marker, Chemicon, Darmstadt, Germany), anti-myeloperoxidase (MPO; 1:1000 dilution, neutrophil marker, Dako, Glostrup, Denmark), anti-endothelial barrier antigen (EBA; 1:1000 dilution, endothelial cell marker, Sternberger Monoclonals, Lutherville, MD, USA), anti-P-glycoprotein (P-gp; 1:5000 dilution, membranetransporter marker, Becton Dickinson, USA), anti-claudin-5 (CLN-5; 1:500 dilution, tight junction protein marker, Invitrogen, Waltham, MA, USA), anti-endothelial nitric oxide synthase (eNOS, 1:2500 dilution, BD Bioscience, Mississauga, ON, Canada), anti-IL-10 (1:200 dilution, anti-inflammatory marker, Santa Cruz, Santa Cruz, CA, USA), anti-IL-1b (1:200 dilution, inflammatory marker, Santa Cruz), anti-CD 31(1:200 dilution, endothelial cell marker, Nouves), anti- aquaporin 4 (AQP-4; 1:50 dilution, astrocyte endfeet marker, Santa Cruz, Santa Cruz, CA, USA) and mouse anti-nuclear mitotic apparatus protein (NuMA, 1:100 dilution; Calbiochem, San Diego, CA, USA), as well as FITClabeled tomato lectin (1:1000; Vector Laboratories, Burlingame, CA, USA).

\section{Animal studies}

Male Sprague-Dawley rats (250-270 g) were anesthetized with $10 \%$ chloral hydrate prior to lipopolysaccharide (LPS) injection. LPS ( $5 \mu \mathrm{g}$ dissolved in $3 \mu \mathrm{l}$ ) was delivered unilaterally into the left substantia nigra (SN; $5.3 \mathrm{~mm}$ posterior, $2.3 \mathrm{~mm}$ lateral, $7.7 \mathrm{~mm}$ ventral from the bregma) and injected at a rate of $0.2 \mu \mathrm{l} /$ minute using a 26-gauge Hamilton syringe attached to an automated microinjector using a stereotaxic apparatus [13]. The needle was then left in place for an additional 10 minutes before slow retraction. The control group was injected with phosphatebuffered saline (PBS; $3 \mu \mathrm{l}$ ) at the same position and using the same method. At 4 hours after LPS injection, MSCs $\left(1 \times 10^{6}\right.$ cells in $\left.200 \mu \mathrm{l}\right)$ were injected into the tail vein [11]. For tissue preparation, rats were sacrificed at 6 hours, 12 hours, 1 day, 2 days, and 7 days after the LPS injection [14]. Histopathological samples from three groups of rats (control, LPS only, MSC treatment following LPS) were compared. The animal work was approved by the Institutional Animal Care and Use Committee of Yonsei University (approval number: 2013-0379).

\section{Isolation of MSCs}

Bone marrow aspirates $(10 \mathrm{ml})$ were obtained from the iliac crests of human donors. The mononuclear cell layer was isolated by Ficoll-Hypaque, washed in PBS, plated in polystyrene plastic $100 \mathrm{~mm}$ culture dishes, and cultivated in low-glucose Dulbecco's modified Eagle's medium (DMEM; Gibco-BRL, Grand Island, NY, USA) containing $10 \%$ fetal bovine serum (FBS; Hyclone, Irvine, CA, USA) and $1 \%$ penicillin/streptomycin (Sigma, St. Louis, MO, USA) in a humidified incubator at $37{ }^{\circ} \mathrm{C}$ under $5 \% \mathrm{CO}_{2}$. Nonadherent cells were removed after 24 hours. When these primary cultures reached $80 \%$ confluence, the cells were harvested using $0.25 \%$ trypsin and subcultured. At passage 6, MSCs were injected into the tail vein or cultured in individual wells of 24-transwell plates $\left(3 \times 10^{3}\right.$ cells/transwell $)$ or six-transwell plates $\left(3 \times 10^{4}\right.$ cells/transwell $) 1$ day before the experiment. The study protocol and consent form were approved by the Institutional Review Board for Human Investigation of Yonsei University Severance Hospital.

\section{Extravasation of Evans blue dye}

To measure the vascular permeability in the brain, Evans blue dye (EB, $4 \%$ in saline, $3 \mathrm{ml} / \mathrm{kg}$ ) was intravenously injected. Fifteen minutes later, the rats were anesthetized and perfused. The frozen sections were prepared, and the infiltrated EB was analyzed using a confocal microscope (Carl Zeiss, Oberkochen, Germany).

\section{Tissue preparation}

For immunohistochemistry, rats were perfused with saline solution containing $0.5 \%$ sodium nitrate and heparin (10 
$\mathrm{U} / \mathrm{ml}$ ) and fixed with $4 \%$ paraformaldehyde dissolved in $0.1 \mathrm{M}$ PBS ( $150 \mathrm{ml} /$ rat$)$ at 12 hours and 7 days after the first drug injection. Brains were removed and post-fixed overnight in buffered $4 \%$ paraformaldehyde at $4{ }^{\circ} \mathrm{C}$ and stored in a $30 \%$ sucrose solution for $1-2$ days at $4{ }^{\circ} \mathrm{C}$ until they sank. The brains were then sectioned into $30 \mu \mathrm{m}$ coronal slices, which were stored in tissue stock solution (30\% glycerol, $30 \%$ ethylene glycol, $30 \%$ three-times distilled water, $10 \% 0.2 \mathrm{M}$ phosphate buffer $(\mathrm{PB}))$ at $4{ }^{\circ} \mathrm{C}$ until required. For western blotting, rats were euthanized at 12 hours after the first injection, and the $\mathrm{SN}$ were rapidly dissected and frozen at $-70{ }^{\circ} \mathrm{C}$. For enzyme-linked immunosorbent assay (ELISA), rats were euthanized at 6 hours, 12 hours, 1 day, 2 days, and 7 days after first injection, and the SN were rapidly dissected and frozen at $-70{ }^{\circ} \mathrm{C}$.

\section{Immunohistochemistry and immunocytochemistry}

Coronal brain sections and cocultured bEnd. 3 cells were rinsed twice in PBS, incubated in $0.2 \%$ Triton X-100 for 30 minutes at room temperature, and rinsed three times with $0.5 \%$ bovine serum albumin (BSA) in $1 \times$ PBS for blocking. After blocking, they were incubated overnight at $4{ }^{\circ} \mathrm{C}$ with primary antibody (anti-TH, anti-P-gp, anti$\mathrm{MPO}$, or anti-EBA), rinsed three times in $1 \times$ PBS containing $0.5 \%$ BSA (10 minutes/rinse), and incubated with the appropriate biotinylated secondary antibody and avidin-biotin complex (Elite Kit; Vector Laboratories, Burlingame, CA, USA) for 1 hour at room temperature. Bound antibodies were visualized by incubating with $0.05 \%$ diaminobenzidine- $\mathrm{HCl}$ (DAB) and $0.003 \%$ hydrogen peroxide in $1 \times$ PBS. Brain sections were rinsed with $1 \times$ PBS for DAB inhibition. Immunostained cells were analyzed by bright-field microscopy.

\section{Immunofluorescence}

Expression of Iba-1, eNOS, CD31, GFAP, AQP-4, IL-10, IL-1 $\beta$, and CLN-5 was assessed using fluorescently tagged secondary antibodies. Brain sections and cocultured bEnd. 3 cells were treated as already described, except that secondary antibodies included goat anti-mouse IgG (Alexa Fluor-488, green) and goat anti-rabbit IgG (Alexa Fluor-594, red). For tomato lectin (TL) labeling, sections were incubated with Alexa Fluor-594conjugated secondary antibodies and FITC-labeled TL for 1 hour. Following secondary antibody incubation, samples were washed and mounted using a Prolong Antifade Kit (Molecular Probes, Waltham, MA, USA). Stained cells and tissues were viewed using an Olympus $\mathrm{I} \times 71$ confocal laser scanning microscope (Olympus, Tokyo, Japan).

\section{bEnd. 3 cell culture}

bEnd. 3 cells (endothelial cell lines; ATCC, University Boulevard Manassas, VA, USA) were maintained in DMEM supplemented with $10 \%$ FBS. Cells were cultured in $25 \mathrm{~cm}^{2}$ flasks and routinely passaged using trypsin-ethylenediamine tetraacetic acid (EDTA) solution. Cells were subcultured into individual wells of 24 -well plates $\left(1 \times 10^{5}\right.$ cells/well $)$ or sixwell plates $\left(1 \times 10^{6}\right.$ cells/well $) 1$ day before the experiment.

\section{Primary astrocyte culture}

Astrocyte was cultured from the cerebral cortices of mouse pups. The cortices were rinsed twice in minimum essential medium (MEM; Sigma) containing $10 \%$ FBS and triturated mechanically. The dissociated cells were plated with $75 \mathrm{~cm}^{2}$ T-flasks. After 2 weeks, the astrocyte was detached from the flasks, and seeded onto 24-well plates $\left(1 \times 10^{5}\right.$ cells/well $)$ or six-well plates $\left(1 \times 10^{6}\right.$ cells/well $)$. After 30 minutes to 1 hour, the culture medium was replaced with MEM containing $5 \%$ FBS.

\section{BV 2 cell culture}

BV 2 cells (microglia lines; ATCC) were maintained in DMEM supplemented with $10 \%$ FBS. Cells were cultured in $25 \mathrm{~cm}^{2}$ flasks and routinely passaged using trypsin-EDTA solution. Cells were cocultured into individual wells of 24 -well plates $\left(5 \times 10^{4}\right.$ cells/well $)$ or six-well plates $\left(5 \times 10^{5}\right.$ cells/well $)$ in seeded astrocytes.

\section{VEGF-A or LPS treatment and coculture with MSCs}

Seeded bEnd.3 cells were treated with VEGF-A $(10 \mathrm{ng} / \mathrm{ml}$; R\&D systems, Minneapolis, MN, USA) in a humidified incubator at $37{ }^{\circ} \mathrm{C}$ for 24 hours. MSCs $\left(3 \times 10^{4}\right.$ cells/well in 24-well plate or $3 \times 10^{5}$ cells/well in six-well plate) in transwells $(0.4 \mu \mathrm{m}$ pore size; Corning, N.Y.C, NY, USA) were then placed in each well and incubated for another 24 hours. Cocultured astrocytes and BV 2 cells were treated with LPS (100 ng/ml; Sigma, Dorset, SP, USA) for 4 hours in a humidified incubator at $37^{\circ} \mathrm{C}$. MSCs $\left(3 \times 10^{4}\right.$ cells/well in 24-well plate or $3 \times 10^{5}$ cells/well in six-well plate) in transwells were then placed in each well and incubated for another 24 hours.

\section{Western blot analysis}

Cocultured bEnd. 3 cells were dissolved in ice-cold lysis buffer (20 mM Tris-HCl, pH 7.5, 1 mM EDTA, $5 \mathrm{mM} \mathrm{MgCl}_{2}$, $1 \mathrm{mM}$ dithiothreitol, $0.1 \mathrm{mM}$ phenylmethylsulfonyl fluoride plus protease inhibitor cocktail; Sigma). Lysates were centrifuged (20 minutes, $14,000 \times g, 4{ }^{\circ} \mathrm{C}$ ) and supernatants were transferred to fresh tubes. Proteins were analyzed using the Bio-Rad Protein Assay Kit (Bio-Rad, Hercules, CA, USA). Equal amounts of protein $(20-50 \mu \mathrm{g})$ were loaded in each lane with loading buffer containing $0.125 \mathrm{M}$ Tris- $\mathrm{HCl}, \mathrm{pH}$ 6.8, 20 \% glycerol, $4 \%$ sodium dodecyl sulfate, $10 \%$ mercaptoethanol, and $0.002 \%$ bromophenol blue. Samples were boiled for 5 minutes before gel loading. Separated proteins were electrophoretically transferred to polyvinylidene difluoride membranes (Millipore, Bedford, MA, USA). Membranes were washed in Tris-buffered 
saline solution with $2.5 \mathrm{mM}$ EDTA (TNE) and then blocked in TNE containing $5 \%$ skim milk for 1 hour. Membranes were incubated overnight at $4{ }^{\circ} \mathrm{C}$ with primary antibodies (CLN-5, eNOS, IL-10, IL-1 $\beta$, VEGF-A) and then were washed three times. The membranes were incubated with secondary antibody (1:10,000 dilution of horseradish peroxidase-conjugated goat anti-rabbit antibody; GenDEPOT, Barker, TX, USA) and anti-mouse antibody (GenDEPOT, Barker, TX, USA) for 1 hour at room temperature. They were developed using ECL western blotting detection reagent (Amerxham, Pittsburgh, PA, USA), followed by exposure to X-ray film. For semiquantitative analysis, the density of immunoblot bands was measured using computer imaging (Fujifilm, Tokyo, Japan).

\section{VEGF-A ELISA}

VEGF-A levels in SN tissue were measured using a sandwich ELISA kit according to the manufacturer's instructions (Abnova, Taipei City, Taiwan). To analyze the changes of VEGF-A levels in the midbrain following MSC treatment, we measured VEGF-A levels at 6 hours, 12 hours, 1 day, 2 days, and 7 days after LPS injection.

\section{Behavior test}

At 1 day and 7 days after LPS injection, apomorphineinduced ( $0.5 \mathrm{mg} / \mathrm{kg}$ subcutaneously; Sigma) rotation was assessed for 30 minutes. Only animals showing a mean of six contralateral rotations/minute over 30 minutes following the administration of apomorphine were retained.

\section{Stereological cell counts}

Unbiased stereological estimations of the total number of $\mathrm{TH}$-positive and MPO-positive cells in the $\mathrm{SN}$ were made using an optical fractionator as described previously, with some modifications [15]. This sampling technique is not affected by tissue volume changes and does not require reference volume determinations [16]. The sections used for counting (eight or nine per series) covered the entire $\mathrm{SN}$ from the rostral tip of the pars compacta to the caudal end of the pars reticulata. Sampling was performed using the Olympus CAST-Grid system (Olympus Denmark A/S, Ballerup, Denmark) in which an Olympus BX51 microscope measured distances on the $z$ axis. The $\mathrm{SN}$ was delineated at $1.25 \times$ magnification. A counting frame $(60 \%, 35$ units, $650 \mu \mathrm{m}^{2}$ ) was placed randomly on the first counting area and systematically moved through all counting areas until the entire delineated area was sampled. Actual counting was performed using a $40 \times$ oil objective. Guard volumes $(4 \mu \mathrm{m}$ from the top and $4-6 \mu \mathrm{m}$ from the bottom of the section) were excluded from both surfaces, and only profiles that came into focus within the counting volume (with a depth of $10 \mu \mathrm{m}$ ) were counted. The total number of stained cells was calculated according to the optical fractionator formula [16].

\section{Statistical analysis}

Mann-Whitney and Kruskal-Wallis tests were used to compare means between groups and for multiple comparisons, respectively. $p<0.05$ was considered statistically significant. Statistical analyses were performed using commercially available software (version 10.0; SPSS, Inc., Chicago, IL, USA).

\section{Results}

\section{MSC treatment reduces BBB permeability in LPS-induced} animals

To determine the effects of MSCs on BBB permeability, brain tissue in the $\mathrm{SN}$ was stained with $\mathrm{EB}$, a marker for $\mathrm{BBB}$ permeability, and antibodies against EBA, a marker of endothelial cells. LPS administration into the SN increased EB infiltration and decreased EBA-positive cells (Fig. 1a, b); however, MSC treatment following LPS administration markedly decreased EB infiltration (Fig. 1a) and increased EBA-positive cells (Fig. 1b). Stereological analysis revealed that MSC treatment significantly increased the number of EBA-positive cells in the SN at 12 hours and 7 days after LPS injection relative to LPStreated rats $(p<0.001$; Fig. 1c). In addition, LPS administration caused a marked increase in P-gp-positive cells (Fig. 1d); however, MSC treatment following LPS administration significantly reduced this effect (Fig. 1d). Quantification confirmed that MSC treatment significantly decreased P-gp-positive cells in the SN of LPS-treated animals compared with only LPS-treated animals at 12 hours and 7 days after LPS injection (Fig. $1 \mathrm{e} ; p<0.001$ ).

\section{MSC treatment enhances filament density in astrocytic endfeet in LPS-induced animals}

The astrocytic endfeet that engulf the capillary networks of the brain have been assumed to significantly influence neurovascular structure and integrity. LPS administration in the SN reduced the density of astrocyte filaments; however, MSC treatment following LPS administration markedly increased the astrocytic filament density at 12 hours and 7 days after LPS injection (Fig. 2a). To further evaluate astrocytic endfeet around vessels, GFAP and TL (Fig. 2a) or AQP-4 and CD31 (Fig. 2b) double staining was performed. Compared with controls, astrocytic filaments and endfeet were sparsely distributed in the SN of LPS-treated animals. On the other hand, MSC treatment markedly increased the density of astrocytic filaments around vessels (Fig. 2a) and astrocytic endfeet (Fig. 2b) in LPS-treated animals. The density of astrocytic filaments around vessels at 7 days after LPS injection was decreased compared with the density at 12 hours after LPS injection. However, MSC treatment increased the density of astrocytic filaments at 7 days after LPS injection relative to the density at 12 hours after LPS injection (Fig. 2a, b). 

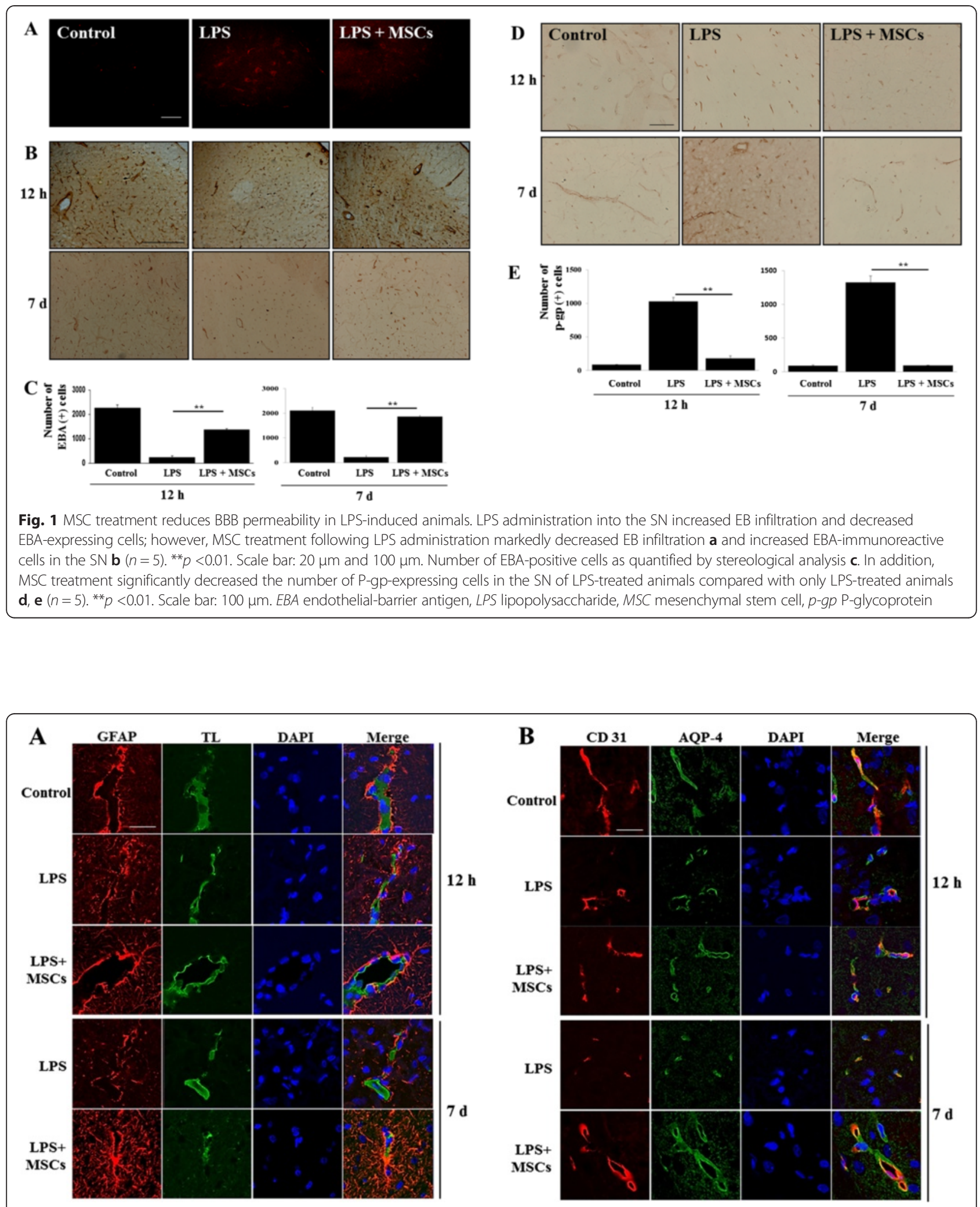

Fig. 2 MSC treatment enhances filament density in astrocytic endfeet in LPS-induced animals. In GFAP and TL a or in AQP-4 and CD31 b double staining, astrocytic filaments and endfeet were sparsely distributed in the SN of LPS-treated animals. On the other hand, MSC treatment markedly increased the density of astrocytic filaments around vessels $\mathbf{a}$ as well as astrocytic endfeet $\mathbf{b}$ in LPS-treated animals. Scale bar: $20 \mu \mathrm{m}$. AQP-4 aquaporin 4, DAPI 4',6-diamidino-2-phenylindole, GFAP glial fibrillary acidic protein, LPS lipopolysaccharide, MSC mesenchymal stem cell, TL tomato lectin 
MSCs modulate tight junction protein expression via VEGF-A in LPS-induced animals

To evaluate the effects of MSCs on expression of VEGF-A, we determined VEGF-A levels by ELISA at 6 hours, 12 hours, 1 day, 2 days, and 7 days after LPS injection. Compared with controls, LPS-treated rats expressed significantly greater levels of VEGF-A from 12 hours after LPS injection, and elevated VEGF-A levels were sustained 7 days after LPS injection. However, MSC treatment significantly reduced VEGF-A expression compared with that in LPS only-treated rats, and these levels were similar to those in controls (Fig. 3a). Furthermore, we evaluated whether the effects of MSCs on VEGF-A regulate eNOS expression and tight junction integrity by double immunostaining for eNOS and CD31 or CLN-5 and CD31. LPS treatment markedly upregulated eNOS immunoreactivity and downregulated CLN-5 immunoreactivity in the endothelial cells of the SN; however, MSC treatment in LPStreated animals attenuated eNOS expression and increased CLN-5 expression in the endothelial cells at 12 hours and 7 days after LPS injection (Fig. 3b, c).

\section{MSC treatment reduces neutrophil infiltration and loss of} dopaminergic cells in the $\mathrm{SN}$

To determine the effects of MSCs on neutrophil infiltration, SN tissue was immunostained for MPO, a marker

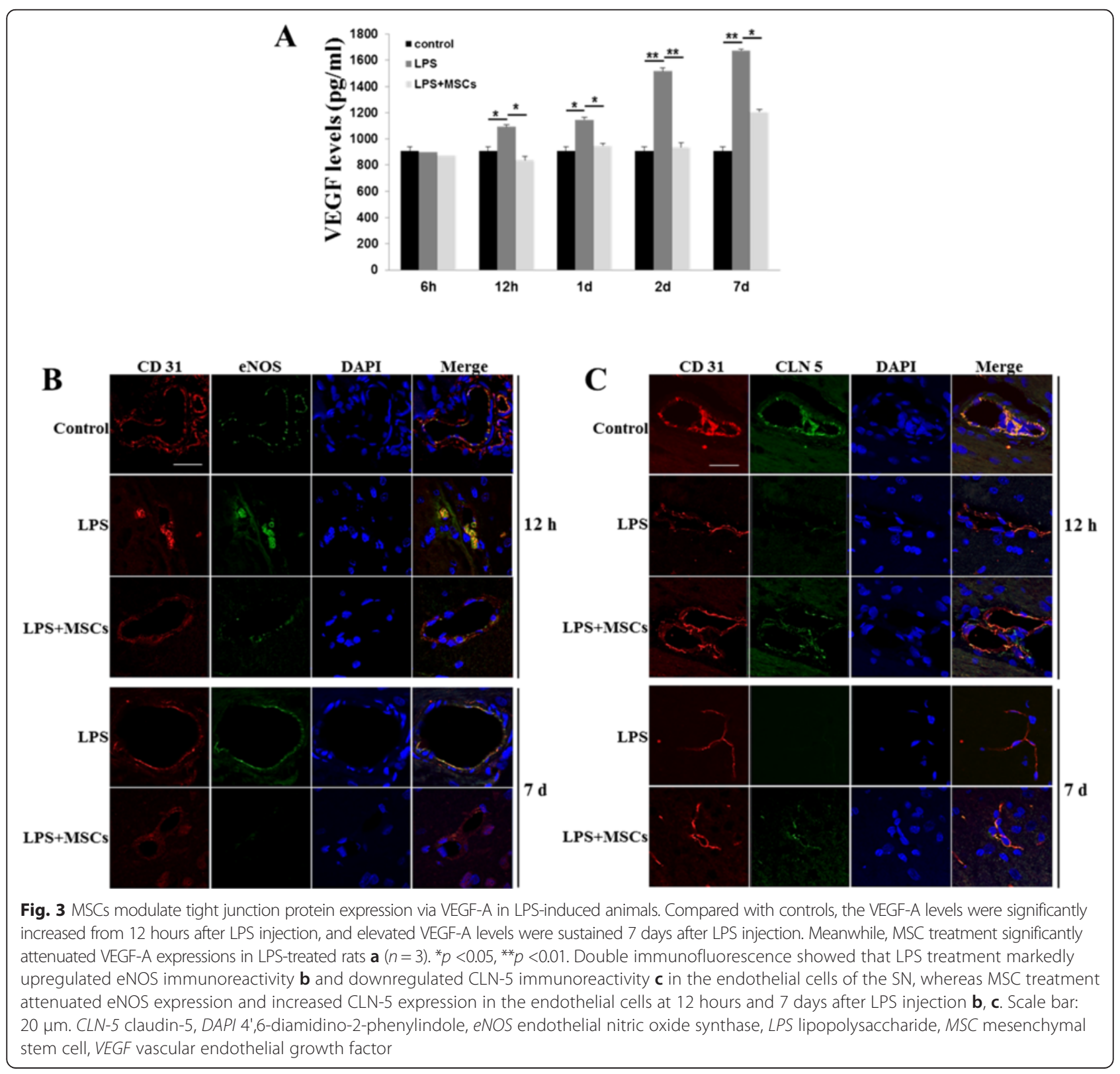



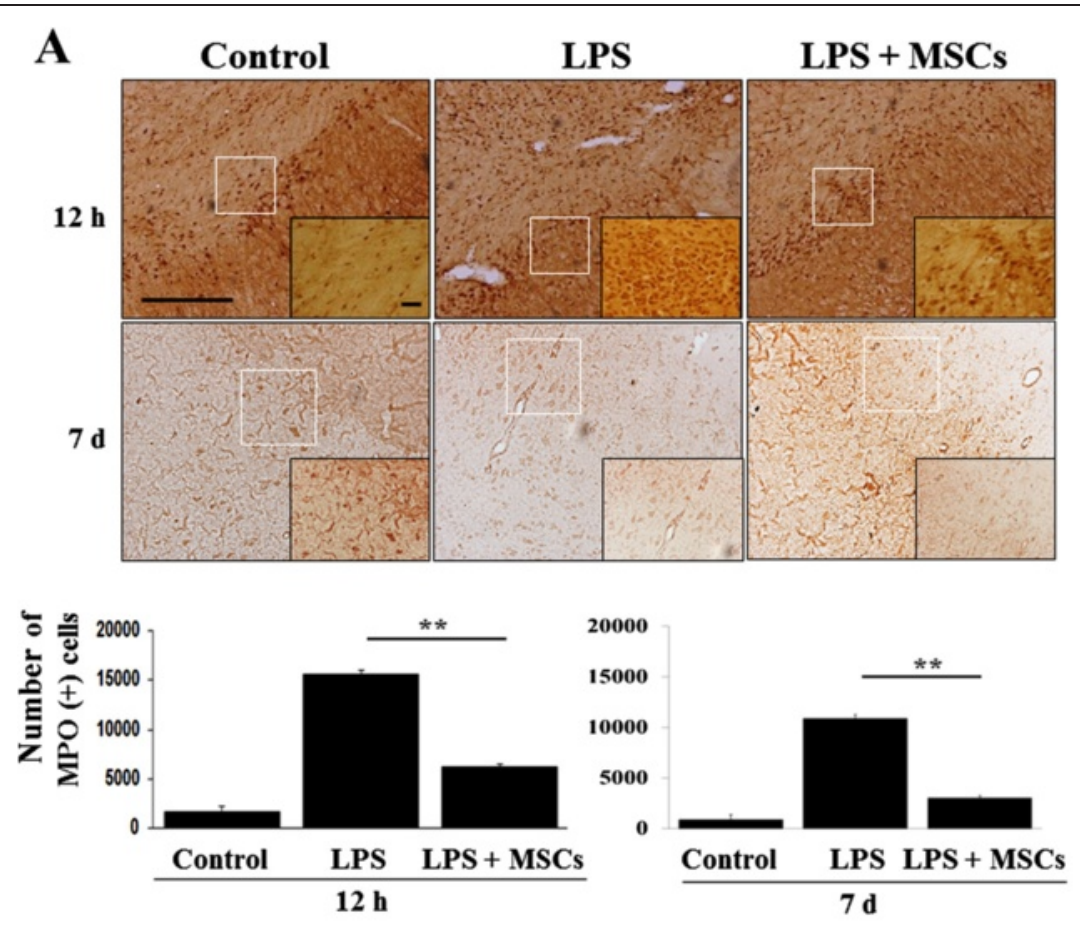

B
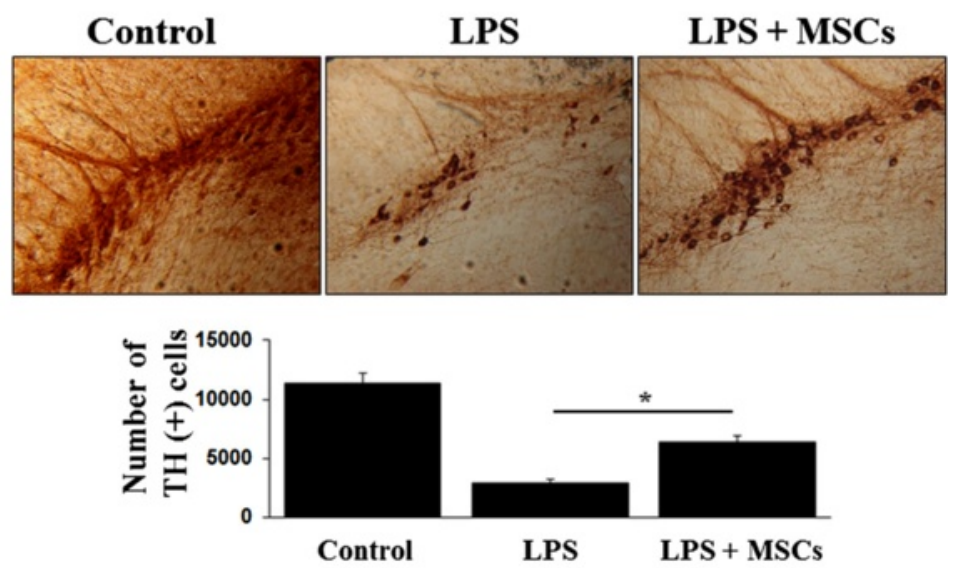

C

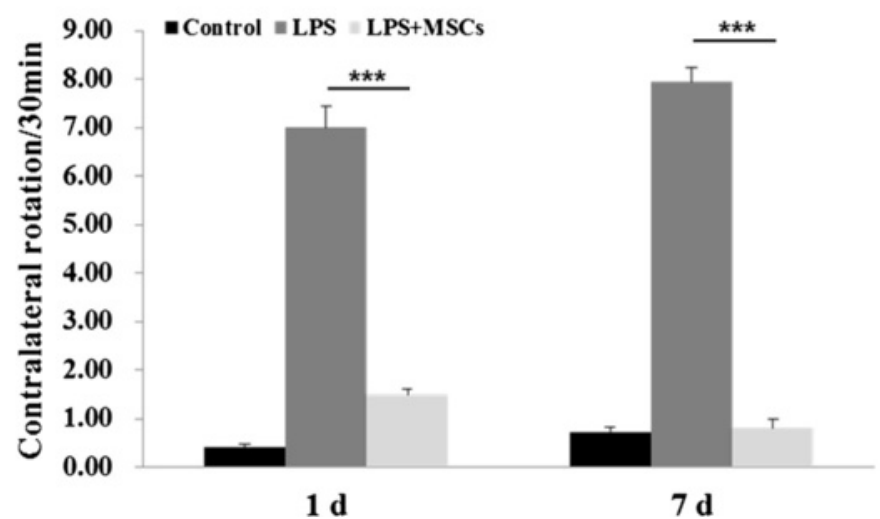

Fig. 4 (See legend on next page.) 
(See figure on previous page.)

Fig. 4 MSC treatment reduces LPS-induced neutrophil infiltration and loss of dopaminergic cells in the midbrain. There was a marked increase in MPO-positive cells in LPS-induced animals; however, MSC treatment following LPS administration notably decreased the number of these cells $\mathbf{a}(n=5)$. ${ }^{* *} p<0.001$. Scale bar: $100 \mu \mathrm{m}$. Consequently, MSC treatment inhibited LPS-induced loss of TH-positive cells in the midbrain $\mathbf{b}(n=5)$. ${ }^{*} p<0.05$. Behavioral analysis showed that the number of rotations after apomorphine injection was significantly increased in animals receiving LPS compared with controls, whereas MSC administration led to a significant reduction of rotation behavior in LPS-treated animals $\mathbf{c}(n=5)$. ${ }^{* *} p<0.001$. Scale bar: $100 \mu \mathrm{m}$. LPS, lipopolysaccharide, MSC mesenchymal stem cell, MPO myeloperoxidase, TH tyrosine hydroxylase

for activated neutrophils. There was a marked increase in MPO-positive cells in LPS-induced animals; however, MSC treatment following LPS administration notably decreased these cells (Fig. 4a). Stereological analysis revealed that the number of neutrophils in the $\mathrm{SN}$ was significantly decreased in MSC-treated rats relative to those treated only with LPS ( $p<0.001$; Fig. 4a). Consequently, MSC treatment decreased LPS-induced loss of dopaminergic neurons (Fig. 4b). On stereological analysis, the number of $\mathrm{TH}$-positive cells in the $\mathrm{SN}$ was significantly increased in MSC-treated rats relative to that in rats treated only with LPS ( $p<0.05$; Fig. $4 \mathrm{~b})$. Behavioral analysis showed that the number of rotations after apomorphine injection was significantly increased in animals receiving LPS compared with controls. However, MSC administration in LPS-treated animals led to a significant reduction of rotation behavior at 1 day and 7 days after LPS injection (Fig. 4c). We attempted to identify MSCs in the MSC-treated animals using humanspecific NuMA immunostaining. The histological analysis showed that NuMA-positive cells were observed in the left SN (see Additional file 1).

\section{MSCs modulate VEGF-A-regulated tight junction protein expression in bEnd. 3 cells}

To determine the effects of MSCs on VEGF-A-related signaling in a cellular system, endothelial cells were treated with VEGF-A and then co-cultured with MSCs. Western blots revealed that VEGF-A treatment significantly increased eNOS expression and decreased CLN-5 expression in bEnd. 3 cells; however, the expressions of these proteins were reverted to control levels after MSC treatment (Fig. 5a). This result was further confirmed by immunocytochemical analysis, which showed that MSC treatment attenuated VEGF-A-induced increases in eNOS immunoreactivity and downregulation of CLN-5 immunoreactivity (Fig. 5b, c). Additionally, we assessed survival of endothelial cells after VEGF-A treatment using EBA immunocytochemistry. Whereas VEGF-A treatment decreased EBA immunoreactivity, MSC treatment in these cells markedly increased EBA-positive cells (Fig. 5d). Stereological analysis revealed that the number of detected EBA-positive cells was significantly increased in MSC-treated cells relative to those treated only with VEGF-A ( $p<0.001$; Fig. 5d).

\section{MSCs modulate VEGF-A through anti-inflammatory action} of microglia

To determine the effects of MSCs on the modulation of VEGF-A in the cellular inflammation system, cocultured astrocyte and BV 2 cells were treated with LPS and then cocultured with MSCs. Western blots revealed that LPS treatment significantly increased IL-1 $\beta$ expression in BV 2 cells and VEGF-A expression in astrocytes; however, the expressions of these proteins were reverted to control levels after MSC treatment (Fig. 6a). In addition, IL10 levels in cocultured astrocytes and BV 2 cells were significantly increased after MSC treatment (Fig. 6a). This result was further confirmed by immunofluorescence analysis, demonstrating that MSC treatment attenuated LPS-induced increases in immunoreactivities of IL-1 $\beta$ and VEGF-A (Fig. 6b, c).

\section{Discussion}

The present study demonstrates that MSC treatment significantly increases survival of dopaminergic neurons by stabilizing $\mathrm{BBB}$ permeability and attenuating neutrophil infiltration in the SN of LPS-injected animals. Furthermore, we found that MSC treatment restores astrocytic endfeet and inhibits VEGF-A-related eNOS-dependent downregulation of CLN-5 in the inflammatory conditions of cellular and animal models. The present data therefore suggest that modulation of astrocytic endfeet and astrocyte-related angiogenic factors by MSCs may represent a therapeutic strategy to stabilize $\mathrm{BBB}$ integrity in neurodegenerative conditions.

Ample evidence has suggested that a microglial reaction and inflammatory processes contribute to the cascade of neuronal degeneration in neurodegenerative disease [17]. Like other central nervous system diseases such as multiple sclerosis, brain cancer, or stroke, neuroinflammation is the main contributor to $\mathrm{BBB}$ dysfunction in neurodegenerative conditions [1, 18-20]. Several clinical studies have demonstrated BBB dysfunction in patients with neurodegenerative diseases; Skoog et al. [21] identified BBB alterations before the onset of clinical dementia in AD patients, and Kortekaas et al. [22] reported BBB impairments in restricted areas of the midbrain in patients with late-stage PD. Regarding the functional significance of BBB dysfunctions, baseline BBB integrity was closely coupled with changes in neurological deficits in patients with multiple system atrophy [23]. Furthermore, in a longitudinal study 

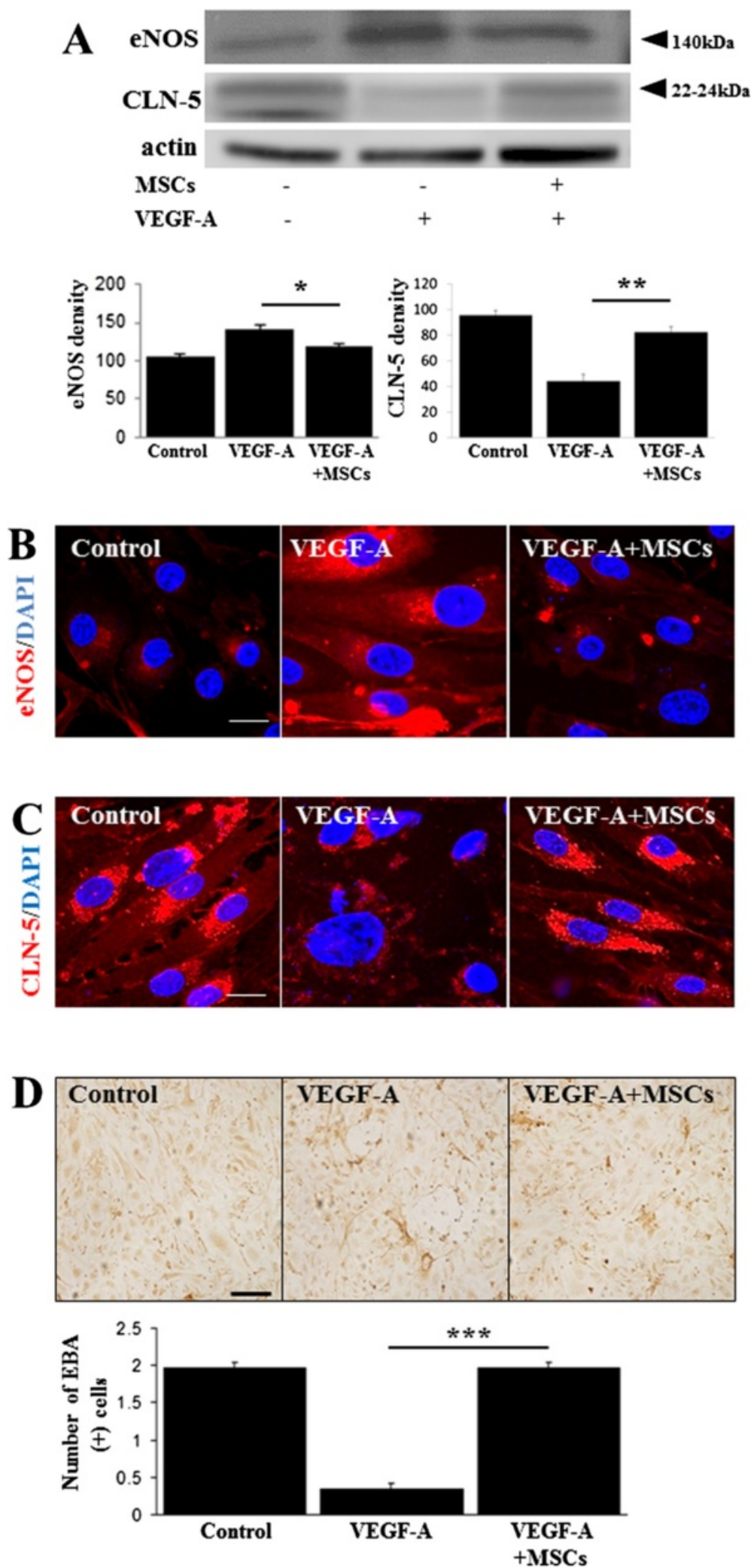

Fig. 5 (See legend on next page.) 
(See figure on previous page.)

Fig. 5 MSCs modulate VEGF-A-regulated tight junction protein expression in bEnd.3 cells. Western blots revealed that VEGF-A treatment significantly increased eNOS expression and decreased CLN-5 expression in bEnd.3 cells; however, the expressions of these proteins were reverted to control levels after MSC treatment $\mathbf{a}(n=3)$. ${ }^{*} p<0.05,{ }^{* *} p<0.01$. Immunocytochemical analysis showed that MSC treatment attenuated VEGF-A-induced increases in eNOS immunoreactivity and downregulation of CLN-5 immunoreactivity b, c. Scale bar: $20 \mu \mathrm{m}$. In addition, the number of detected EBA-expressing cells was significantly increased in MSC-treated cells relative to those treated only with VEGF-A d, e $(n=3){ }^{* * *} p<0.001$. Scale bar: $100 \mu \mu m$. CLN-5 claudin-5, DAPI 4',6-diamidino-2-phenylindole, EBA endothelial-barrier antigen, eNOS endothelial nitric oxide synthase, LPS lipopolysaccharide, MSC mesenchymal stem cell, VEGF vascular endothelial growth factor

of multiple system atrophy patients, we recently demonstrated that changes in $\mathrm{BBB}$ integrity may be a more reliable biological marker for disease progression in neurodegenerative disease than is baseline BBB status [24]. Collectively, these in vivo data suggest that $\mathrm{BBB}$ integrity might modify disease progression in neurodegenerative diseases, and thus modulation of BBB integrity could be a potential treatment for neurodegenerative diseases.
The present study demonstrated that MSCs stabilized $\mathrm{BBB}$ alterations induced by LPS administration into the $\mathrm{SN}$ of rats. MSC treatment significantly restored the expression of EBA and P-gp in endothelial cells, which were significantly altered in LPS-treated animals. Consequently, MSC treatment stabilized infiltrations of EB and activated neutrophils, and thus increased the survival of dopaminergic neurons in the midbrain of LPS-treated
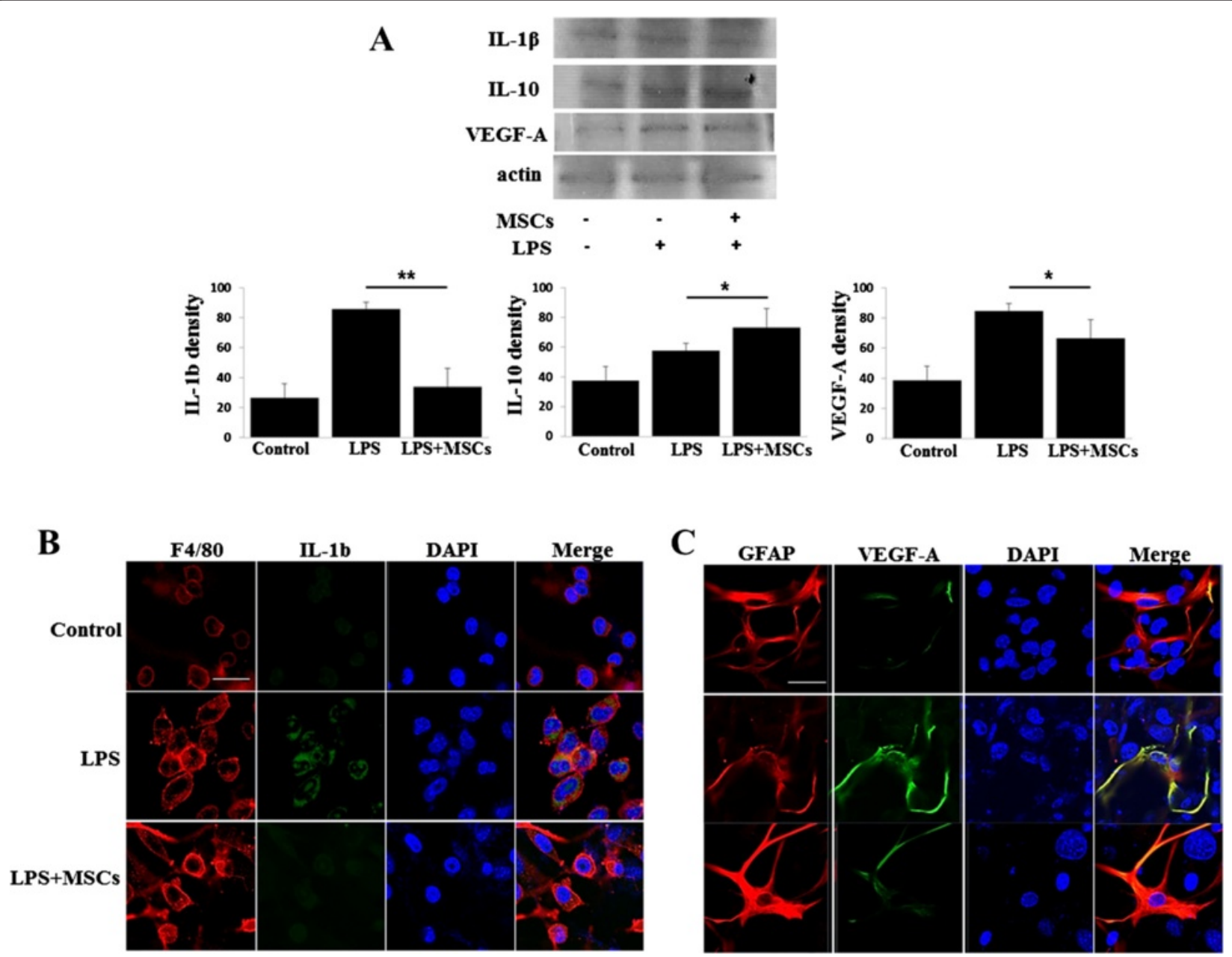

Fig. 6 MSCS modulate VEGF-A through anti-inflammatory action of microglia. Using coculture of astrocyte and BV 2 cells, LPS treatment significantly increased IL-1 $\beta$ expression in BV 2 cells and VEGF-A expression in astrocytes. However, MSC treatment significantly decreased the expressions of these proteins with an increase in IL-10 levels a $(n=3) .{ }^{*} p<0.05,{ }^{* *} p<0.01$. Immunofluorescence analysis showed that MSC treatment attenuated LPS-induced increased in immunoreactivities of IL-1 $\beta$ in BV 2 cells $\mathbf{b}$ and VEGF-A in astrocytes $\mathbf{c}$. Scale bar: $20 \mu \mathrm{m}$. DAPI 4',6-diamidino-2-phenylindole, GFAP glial fibrillary acidic protein, IL interleukin, LPS lipopolysaccharide, MSC mesenchymal stem cell, VEGF vascular endothelial growth factor 
rats. We focused our investigation on the mechanism by which MSCs modulate BBB integrity on astrocytic endfeet and astrocyte-related angiogenic factors. Astrocytes are known to be more resistant than neurons to oxidative stress [25], and they are neuroprotective because they take up potassium and glutamate and release mitogenic factors [26]. However, in the inflamed brain, astrocytes retract their endfeet from vessels, increasing $\mathrm{BBB}$ permeability [4-6]. More importantly, several studies have documented that expression of the angiogenic factor VEGF-A by astrocytes is increased by microgliainduced inflammatory factor IL- $1 \beta$ in the inflamed brain $[7,27,28]$. In turn, VEGF-A released from astrocytes binds to VEGF receptor 2 on microvascular endothelial cells and activates eNOS to downregulate expression of tight junction proteins, which may lead to disruption of endothelial tight junctions and increased BBB permeability $[7,9]$.

Interestingly, the mechanism by which MSCs stabilize the BBB through astrocytes seems to be complex. First, our study demonstrated that MSCs can restore the density of filaments within astrocytic endfeet surrounding microvessels. As astrocytic endfeet are a major component of the BBB, a lower density of astrocytic filaments in the midbrain is known to reduce BBB integrity in LPS-induced models of PD [29]. Restoration of astrocytic endfeet by MSCs may thus represent a morphologically stabilizing effect on BBB integrity. Second, the present study indicates that MSCs may modulate VEGFA-related eNOS-dependent downregulation of tight junction proteins in the endothelium. In LPS-treated rats, MSC treatment decreased LPS-induced elevation of VEGF-A levels and downregulation of CLN-5. In cultured endothelial cells, employed to directly evaluate modulatory effects of MSCs on VEGF-A-related eNOSdependent signaling, MSC treatment reduced VEGF-Ainduced increases in eNOS expression and decreases in CLN-5 expression, which consequently led to increased survival of endothelial cells. Regarding the underlying mechanisms of VEGF-A-related eNOS-dependent signaling by MSCs, inflammatory cytokine IL- $1 \beta$ released by activated microglia would trigger this signaling pathway by binding to its receptor on the astrocyte [9]. Our previous study showed that MSCs could attenuate microglial activation though secretion of the anti-inflammatory cytokines IL-6, IL-10, and transforming growth factor beta in LPS-induced or neurotoxin-induced animal models [11]. In addition, the present study showed that, along with increased IL-10 levels after MSC treatment, MSC treatment significantly decreased the IL- $1 \beta$ level in BV 2 cells and the VEGF-A level in astrocytes that were elevated following LPS treatment. Accordingly, the regulatory property of microglial activation by MSCs might decrease secretion of proinflammatory cytokines into the astrocytes and then modulate sequentially VEGF-A-related eNOS-dependent signaling in the endothelium, which would stabilize expression of tight junction proteins.

The consequence of BBB alteration in parkinsonian diseases remains unclear. In neurodegenerative disease, neurons and glia are confronted with a decreased homeostatic reserve and thus become extremely sensitive and vulnerable to alterations in the extracellular milieu [30]. In turn, increased $\mathrm{BBB}$ permeability may increase the probability of exposing microenvironments in the brain to environmental neurotoxins, which may then interrupt mitochondrial electron transport or elements of the peripheral immune system, and subsequently accelerate neuroglial degeneration and neuroinflammation. BBB stabilization might therefore be an effective strategy to modify disease progression in neurodegenerative disease.

Whether impaired BBB integrity in neurodegenerative conditions is a compensatory response to expel toxic misfolded proteins from the brain remains unclear. Nevertheless, BBB alterations would be expected to further accelerate neuroinflammation as well as neurodegeneration [30]. Similarly, the present study showed that BBB disruption by neuroinflammation led to neutrophil infiltration in addition to dopaminergic neuronal death. However, MSC treatment restored activated neutrophil infiltrations and dopaminergic neuronal death. In this regard, regardless of the underlying response for $\mathrm{BBB}$ alterations, stabilizing the $\mathrm{BBB}$ may represent a useful therapeutic option to prevent and/or modify disease progression in PD.

\section{Conclusions}

We have shown that MSCs stabilize BBB permeability by modulating astrocytic endfeet and VEGF-A signaling, which may be relevant as a candidate neuroprotective strategy for the treatment of neurodegenerative disease.

\section{Additional file}

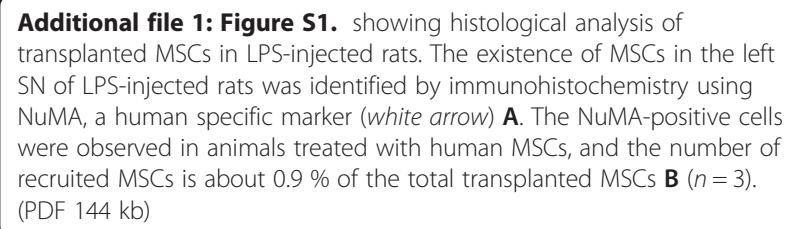

\section{Abbreviations}

AQP-4: Aquaporin 4; BBB: Blood-brain barrier; BSA: Bovine serum albumin; CLN-5: Claudin-5; DAB: $0.05 \%$ diaminobenzidine-HCl; DMEM: Dulbecco's modified Eagle's medium; EB: Evans blue dye; EBA: Endothelial-barrier antigen; EDTA: Ethylenediamine tetraacetic acid; ELISA: Enzyme-linked immunosorbent assay; eNOS: Endothelial nitric oxide synthase; FBS: Fetal bovine serum; FTIC: Fluorescein isothiocyanate; GFAP: Glial fibrillary acidic protein; Iba-1: Ionized calcium-binding adapter molecule 1; IL-10: Interleukin-10; LPS: Lipopolysaccharide; MEM: Minimum essential medium;

MPO: Myeloperoxidase; MPTP: 1-Methyl-4-phenyl-1,2,3,6-tetrahydropyridine; MSC: Mesenchymal stem cell; NuMA: Nuclear mitotic apparatus protein; PBS: Phosphate-buffered saline; PD: Parkinson's disease; P-gp: P-glycoprotein; 
SN: Substantia nigra; TH: Tyrosine hydroxylase; TL: Tomato lectin; TNE: Tris-buffered saline solution with 2.5 mM EDTA; VEGF-A: Vascular endothelial growth factor -A.

\section{Competing interests}

The authors declare that they have no competing interests.

\section{Authors' contributions}

HJP was responsible for conception and design, acquisition of data, analysis and interpretation of the data, and drafting the manuscript. JYS was responsible for discussion of data interpretation. HNK was responsible for discussion of data interpretation, and carried out the cell culture. SHO was responsible for discussion of data interpretation, and carried out the cell culture. SKS was responsible for discussion of data interpretation. PHL was responsible for conception and design, analysis and interpretation of the data, and general supervision of the research. All authors read and approved the final manuscript.

\section{Acknowledgements}

This study was supported by Basic Science Research Program through the National Research Foundation of Korea (NRF) funded by the Ministry of Science, ICT and future Planning (NRF- 2013R1A2A2A01067761).

\section{Author details}

${ }^{1}$ Department of Neurology and Brain Research Institute, Yonsei University College of Medicine, 250 Seongsanno, Seodaemun-gu, Seoul 120-752, South Korea. ${ }^{2}$ Severance Biomedical Science Institute, Yonsei University College of Medicine, 250 Seongsanno, Seodaemun-gu, Seoul 120-752, South Korea. ${ }^{3}$ Department of Neurology, Jeju University College of Medicine, 102 Jejudaehak-ro, Jeju 690-756, South Korea.

\section{Received: 1 May 2015 Revised: 31 August 2015}

\section{Accepted: 7 September 2015 Published online: 29 September 2015}

\section{References}

1. Desai BS, Monahan AJ, Carvey PM, Hendey B. Blood-brain barrier pathology in Alzheimer's and Parkinson's disease: implications for drug therapy. Cell Transplant. 2007;16:285-99.

2. Bowman GL, Kaye JA, Moore M, Waichunas D, Carlson NE, Quinn JF. Blood-brain barrier impairment in Alzheimer disease: stability and functional significance. Neurology. 2007;68:1809-14.

3. Willis CL, Nolan CC, Reith SN, Lister T, Prior MJ, Guerin CJ, et al. Focal astrocyte loss is followed by microvascular damage, with subsequent repair of the blood-brain barrier in the apparent absence of direct astrocytic contact. Glia. 2004;45:325-37.

4. Mani N, Khaibullina A, Krum JM, Rosenstein JM. Astrocyte growth effects of vascular endothelial growth factor (VEGF) application to perinatal neocortical explants: receptor mediation and signal transduction pathways. Exp Neurol. 2005;192:394-406.

5. Ridet JL, Malhotra SK, Privat A, Gage FH. Reactive astrocytes: cellular and molecular cues to biological function. Trends Neurosci. 1997;20:570-7.

6. Walton M, Connor B, Lawlor P, Young D, Sirimanne E, Gluckman P, et al. Neuronal death and survival in two models of hypoxic-ischemic brain damage. Brain Res Brain Res Rev. 1999;29:137-68.

7. Argaw AT, Gurfein BT, Zhang Y, Zameer A, John GR. VEGF-mediated disruption of endothelial CLN-5 promotes blood-brain barrier breakdown. Proc Natl Acad Sci U S A. 2009;106:1977-82.

8. Proescholdt MA, Jacobson S, Tresser N, Oldfield EH, Merrill MJ. Vascular endothelial growth factor is expressed in multiple sclerosis plaques and can induce inflammatory lesions in experimental allergic encephalomyelitis rats. J Neuropathol Exp Neurol. 2002;61:914-25.

9. Argaw AT, Asp L, Zhang J, Navrazhina K, Pham T, Mariani JN, et al. Astrocyte-derived VEGF-A drives blood-brain barrier disruption in CNS inflammatory disease. J Clin Invest. 2012;122:2454-68.

10. Park HJ, Lee PH, Bang OY, Lee G, Ahn YH. Mesenchymal stem cells therapy exerts neuroprotection in a progressive animal model of Parkinson's disease. J Neurochem. 2008;107:141-51.

11. Kim YJ, Park HJ, Lee G, Bang OY, Ahn YH, Joe E, et al. Neuroprotective effects of human mesenchymal stem cells on dopaminergic neurons through anti-inflammatory action. Glia. 2009;57:13-23.

12. Chao YX, He BP, Tay SS. Mesenchymal stem cell transplantation attenuates blood brain barrier damage and neuroinflammation and protects dopaminergic neurons against MPTP toxicity in the substantia nigra in a model of Parkinson's disease. J Neuroimmunol. 2009;216:39-50.

13. Paxinos G, Watson CRR, Emson PC. Ache-stained horizontal sections of the rat-brain in stereotaxic coordinates. J Neurosci Meth. 1980;3:129-49.

14. Ji KA, Eu MY, Kang SH, Gwag BJ, Jou I, Joe EH. Differential neutrophil infiltration contributes to regional differences in brain inflammation in the substantia nigra pars compacta and cortex. Glia. 2008;56:1039-47.

15. Kirik D, Rosenblad C, Bjorklund A. Characterization of behavioral and neurodegenerative changes following partial lesions of the nigrostriatal dopamine system induced by intrastriatal 6-hydroxydopamine in the rat. Exp Neurol. 1998;152:259-77.

16. West MJ, Slomianka L, Gundersen HJ. Unbiased stereological estimation of the total number of neurons in thesubdivisions of the rat hippocampus using the optical fractionator. Anat Rec. 1991;231:482-97.

17. Gao HM, Hong JS. Why neurodegenerative diseases are progressive: uncontrolled inflammation drives disease progression. Trends Immunol. 2008:29:357-65.

18. Bruck W, Bitsch A, Kolenda H, Bruck Y, Stiefel M, Lassmann H. Inflammatory central nervous system demyelination: correlation of magnetic resonance imaging findings with lesion pathology. Ann Neurol. 1997;42:783-93.

19. Dallasta LM, Pisarov LA, Esplen JE, Werley JV, Moses AV, Nelson JA, et al. Blood-brain barrier tight junction disruption in human immunodeficiency virus-1 encephalitis. Am J Pathol. 1999;155:1915-27.

20. Sandoval KE, Witt KA. Blood-brain barrier tight junction permeability and ischemic stroke. Neurobiol Dis. 2008;32:200-19.

21. Skoog I, Wallin A, Fredman P, Hesse C, Aevarsson O, Karlsson I, et al. A population study on blood-brain barrier function in 85-year-olds: relation to Alzheimer's disease and vascular dementia. Neurology. 1998;50:966-71.

22. Kortekaas R, Leenders KL, van Oostrom JC, Vaalburg W, Bart J, Willemsen AT, et al. Blood-brain barrier dysfunction in parkinsonian midbrain in vivo. Ann Neurol. 2005;57:176-9.

23. Song SK, Lee SK, Lee JJ, Lee JE, Choi HS, Sohn YH, et al. Blood-brain barrier impairment is functionally correlated with clinical severity in patients of multiple system atrophy. Neurobiol Aging. 2011;32:2183-9.

24. Lee JE, Song SK, Hong JY, Sunwoo MK, Park HJ, Sohn YH, et al. Changes in the blood-brain barrier status closely correlate with the rate of disease progression in patients with multiple system atrophy: a longitudinal study. Parkinsonism Relat Disord. 2013;19:450-2.

25. Marrif $\mathrm{H}$, Juurlink $\mathrm{BH}$. Astrocytes respond to hypoxia by increasing glycolytic capacity. J Neurosci Res. 1999;57:255-60.

26. Swanson RA. Astrocyte glutamate uptake during chemical hypoxia in vitro. Neurosci Lett. 1992;147:143-6.

27. Bush TG, Puvanachandra N, Horner CH, Polito A, Ostenfeld T, Svendsen CN, et al. Leukocyte infiltration, neuronal degeneration, and neurite outgrowth after ablation of scar-forming, reactive astrocytes in adult transgenic mice. Neuron. 1999;23:297-308.

28. Herx LM, Yong WW. Interleukin-1 beta is required for the early evolution of reactive astrogliosis following CNS lesion. J Neuropathol Exp Neurol. 2001;60:961-71.

29. Tomas-Camardiel M, Rite I, Herrera AJ, de Pablos RM, Cano J, Machado A, et al. Minocycline reduces the lipopolysaccharide-induced inflammatory reaction, peroxynitrite-mediated nitration of proteins, disruption of the blood-brain barrier, and damage in the nigral dopaminergic system. Neurobiol Dis. 2004;16:190-201

30. Popescu BO, Toescu EC, Popescu LM, Bajenaru O, Muresanu DF, Schultzberg $\mathrm{M}$, et al. Blood-brain barrier alterations in ageing and dementia. J Neurol Sci. 2009:283:99-106. 